\title{
Risk factors for uncontrolled asthma in adults
}

\section{Factores de riesgo para mal control de asma en adultos}

\author{
Juan Carlos Fernández de Córdova-Aguirre, ${ }^{1}$ Andrea Aída Velasco-Medina, ${ }^{1}$ César Urquiza,${ }^{1}$ \\ Karol Andrea Guzmán-Guillén, ${ }^{1}$ Guillermo Velázquez-Sámano ${ }^{1}$
}

\begin{abstract}
Background: Many studies in children had identified risk factors associated with partly or uncontrolled asthma. In adults, factors related to asthma control were not well defined.

Objective: To find the factors related to partly or uncontrolled asthma in adults.

Methods: Asthmatic adults who had severity at least in the mild persistent level, were recruited. The asthma control levels were classified as controlled, partly controlled and uncontrolled according to the Global Initiative for Asthma guideline. Risk factors were compared between the patients with controlled and partly controlled/ uncontrolled asthma groups.

Results: two hundred (75.5\% females) were included. The mean age was $38.4 \pm 11.93$ years old. There were 100 patients (50\%) in the controlled and 100 patients (50\%) in partly controlled and uncontrolled asthma group. For asthma severity, 35 patients (88.2 \%) were in mild persistent, 14 patients (10\%) in moderate persistent and 97 patients $(1.8 \%)$ in severe persistent groups. The absence of stress yielded an $\mathrm{OR}=0.41(95 \% \mathrm{Cl}=0.23-0.75), \mathrm{p}=0.03$. While active smoking, $\mathrm{OR}=4.23(95 \% \mathrm{Cl}=1.35-13.2), \mathrm{p}=0.007$. Patients who have no contact with stuffed animals or rugs have OR $=0.4(\mathrm{IC} 95 \%=0.2-0.79)$. It was important the result of the patients that if they correctly use their inhalers OR $=0.36($ IC $95 \%=0.13-0.97), p=0.031$.

Conclusions: Current and active smoking and atopic dermatitis are risk factors. As a protective factor to the normal levels of eosinophils in blood, correct use of inhalers, $\mathrm{FEV}_{1}>80 \%$, the absence of stress and the absence of contact with carpets and stuffed animals.
\end{abstract}

Keywords: Asthma; Adults; GINA

Este artículo debe citarse como: Fernández de Córdova-Aguirre JC, Velasco-Medina AA, Urquiza C, Guzmán-Guillén KA, Velázquez-Sámano G. Factores de riesgo para mal control de asma en adultos. Rev Alerg Mex. 2019;66(1):65-75

ORCID

Juan Carlos Fernández de Córdova-Aguirre, 0000-0002-4788-5868;

Andrea Aída Velasco-Medina, 0000-0002-5215-1906; César Urquiza, 0000-0002-2501-384X;

Karol Andrea Guzmán-Guillén, 0000-0002-1840-4690; Guillermo Velázquez-Sámano, 0000-0002-8247-4300

'Secretaría de Salud, Hospital General de México, Servicio de Alergia e Inmunología Clínica, Ciudad de México, México
Correspondencia: Guillermo Velázquez-Sámano. gvelazquezsamano@yahoo.com

Recibido: 2018-10-03

Aceptado: 2019-01-15

DOI: $10.29262 /$ ram.v66i1.557 


\section{Resumen}

Antecedentes: Estudios en niños han identificado factores asociados con la falta de control del asma. En adultos no están bien definidos.

Objetivo: Identificar los factores relacionados con la falta de control del asma en adultos.

Métodos: Se incluyeron adultos con asma clasificados como controlados, parcialmente controlados y no controlados según los criterios de la Global Initiative for Asthma.

Resultados: Se incluyeron 200 pacientes (75.5 \% mujeres), edad promedio de $38.4 \pm 11.93$ años. Cien pacientes con asma controlada y 100 con asma no controlada y parcialmente controlada; 35 (88.2 \%) con persistencia leve, $14(10 \%)$ con persistencia moderada y $97(1.8 \%)$ con persistencia grave; en estrés se encontró RM = 0.41 (IC $95 \%=0.23-0.75), p=0.03$; en tabaquismo actual y activo, $\mathrm{RM}=4.23$ (IC $95 \%=1.35-13.2), \mathrm{p}=0.007$; $\mathrm{y}$ en uso correcto de inhaladores, $\mathrm{RM}=0.36$ (IC $95 \%=0.13-0.97), p=0.031$. Los pacientes no tuvieron contacto con peluches ni alfombras. Conclusiones: Se observó asociación con tabaquismo actual activo y dermatitis atópica. Como factores de protección se identificó niveles normales de eosinófilos en sangre, uso correcto de inhaladores, $\mathrm{VEF}_{1}>80 \%$, ausencia de estrés y de contacto con alfombras y peluches.

Palabras clave: Asma; Adultos; GINA

\section{Abreviaturas y siglas}

ERGE, enfermedad por reflujogastroesofágico

FEP, flujo espiratorio pico
GINA, Global Initiative for Asthma

VEF $_{1}$, volumen espiratorio forzado en el primer segundo

\section{Antecedentes}

El asma es una enfermedad crónica y común que afecta a niños y adultos en todo el mundo, ${ }^{1}$ con una prevalencia variable que alcanza $44 \%{ }^{2}$ La calidad de vida de los pacientes asmáticos mal controlados es mala, constantemente visitan a su médico, acuden a los servicios de urgencias, presentan ausentismo laboral o escolar, tiene limitaciones para realizar las actividades diarias normales $\mathrm{y}$, además, tienen problemas para descansar por las noches. ${ }^{3}$ Por lo tanto, el objetivo principal del tratamiento no farmacológico y farmacológico del asma es reducir y controlar los signos y síntomas de esta patología, lo cual conlleva a mejorar la calidad de vida de los pacientes, evitar posibles recaídas y de esta manera disminuir costos. ${ }^{4}$ La Global Initiative for Asthma (GINA), recomienda clasificar a los pacientes con asma según el grado de control de la enfermedad en tres niveles o categorías: controlado, parcialmente controlado y no controlado, ${ }^{5}$ y de acuerdo con varios criterios: signos y síntomas diurnos y nocturnos, dificultades para realizar las actividades diarias, la necesidad de utilizar medicamentos de rescate, exacerbaciones y pruebas funcionales respiratorias, la medición del flujo espiratorio pico (FEP), flujometría y del volumen espiratorio forzado en el primer segundo $\left(\mathrm{VEF}_{1}\right)$ mediante espirometría. ${ }^{6}$ Sin embargo, para catalogar la severidad del asma, GINA recomienda clasificarla en cuatro categorías, ${ }^{7}$ considerando signos y síntomas diurnos y nocturnos, $\mathrm{VEF}_{1}$ o FEP: $^{8}$

- Leve intermitente.

- Leve persistente.

- Moderada persistente.

- Severa persistente.

Actualmente se considera que el control de la enfermedad es el mejor predictor de problemas y exacerbaciones en el futuro. ${ }^{9}$ A pesar de los avances en el diagnóstico y tratamiento de esta enfermedad, numerosos pacientes tienen sintomatología que dificulta su vida diaria, incluso presentan agudizaciones graves que requieren hospitalizaciones en los servicios de urgencias. ${ }^{10}$ Los últimos reportes mencionan que $15 \%$ de los japoneses y $19 \%$ de los americanos se clasifican con asma severa persistente. ${ }^{11}$ En Europa, 
solo $15 \%$ de los asmáticos que utilizan esteroides inhalados a dosis altas están bien controlados. ${ }^{12}$

Entre los principales factores de mal control del asma están el uso de esteroides orales, comorbilidades crónicas, alteración de base en las pruebas funcionales, asma severa al momento del diagnóstico, exposición a factores desencadenantes ambientales, infecciones y bajo nivel escolar. ${ }^{11,12}$ Existen varios estudios que han mostrado los posibles factores de riesgo para un mal control del asma, especialmente en niños. En México existe poco conocimiento en cuanto a factores de riesgo asociados con mal control del asma en adultos. El objetivo de este estudio es describir las causas del mal control del asma en este grupo poblacional.

\section{Métodos}

Diseño observacional, longitudinal, comparativo, de casos y controles. Fue presentado y aprobado por la Comisión de Investigación y Ética del Hospital General de México, Ciudad de México, con clave de registro DI/14/309/03/027. La población estudiada estuvo constituida por pacientes asmáticos adultos diagnosticados por un alergólogo conforme a los criterios establecidos en GINA, que acudieron al Servicio de Alergia e Inmunología Clínica de ese hospital. Se formaron dos grupos: casos $(\mathrm{n}=100)$ y controles $(n=100)$ :

- Casos, pacientes asmáticos mayores de 18 años que en el último mes antes de la entrevista reunieran uno o más de los siguientes criterios de control: signos y síntomas de asma durante el día, despertares nocturnos, necesidad de uso de medicamentos de rescate como salbutamol o bromuro de ipratropio, dificultad para realizar las actividades cotidianas diariamente o con los valores de la flujometría o del $\mathrm{VEF}_{1}$ mediante espirometría $<80 \%$ de lo normal.

- Controles, pacientes asmáticos mayores de 18 años que en el último mes antes de la entrevista no presentaron criterios de mal control, es decir, que no tengan signos ni síntomas de asma durante el día, que no presentaran despertares nocturnos, que no hayan utilizado medicación de rescate como salbutamol o bromuro de ipratropio, que no tuvieran dificultad para realizar las actividades cotidianas diariamente y con los valores de la flujometría o del $\mathrm{VEF}_{1}$ mediante espirometría $>80 \%$.
Los criterios de inclusión fueron pacientes mayores a 18 años de edad, de primera vez y subsecuentes, con diagnóstico de asma de acuerdo con los criterios de GINA, historia clínica completa con estudios de laboratorio y que firmaron el consentimiento informado. Los criterios de exclusión fueron inmunodeficiencias primarias y secundarias, enfermedades cardiacas (insuficiencia cardiaca congestiva, asma cardiaca, cor pulmonale crónico), enfermedad pulmonar obstructiva crónica, asma por embarazo, aspergilosis broncopulmonar alérgica, neumonitis intersticial y tuberculosis pulmonar. Los criterios de eliminación fueron pacientes de quienes no se dispusiera de los datos completos sobre las variables en estudio o que expresaron su voluntad de retirarse del estudio.

\section{Procedimientos}

A los sujetos de estudio se les aplicó el protocolo diagnóstico y terapéutico para pacientes asmáticos. Se realizó historia clínica y examen físicos detallados, estudios complementarios de sangre, coproparasitoscopia, rayos $\mathrm{X}$ de tórax, espirometría basal y posterior al uso de broncodilatador inhalado, así como pruebas cutáneas. La variable independiente fue asma parcialmente controlada o mal controlada y las variables dependientes fueron los factores de riesgo estudiados. El muestreo fue aleatorio, estratificado y pareado por sexo y edad. El tipo de instrumento de medición fue observacional. Se midió peso y talla.

Se realizaron estudios en sangre para determinar los valores de IgE sérica total y los eosinófilos en sangre periférica. Los valores de referencia de los eosinófilos que el laboratorio proporciona son $<450 \mathrm{~mm}^{3}$ y los valores de IgE total $>160 \mu \mathrm{g} / \mathrm{dL}$. Se realizaron pruebas funcionales respiratorias pre y posbroncodilatación con los criterios de GINA y los valores en la población mexicana de acuerdo con Pérez-Padilla. ${ }^{13}$ Se efectuaron e interpretaron las pruebas cutáneas por punción cutánea bajo los criterios de la American Academy of Allergy, Asthma and Immunology, ${ }^{14}$ con diferentes alérgenos (pólenes de árboles, malezas y pastos, hongos, perro, gato, ácaros y cucarachas). La recolección de los datos se realizó a través de una encuesta directa. Se adecuó el cuestionario de Patiño et al., el cual fue realizado en el Servicio de Alergia e Inmunología Clínica del Hospital General y aceptado por la Dirección de Investigación de esa institución. 


\section{Estadística}

Se calculó un tamaño de muestra de 200 sujetos, con una potencia de $80 \%$ para detectar una diferencia entre las proporciones de 0.10 (delta). Se asumió que la proporción en el grupo 1 fue de 0.27 bajo hipótesis nula y de 0.37 bajo hipótesis alternativa. El estadístico fue la prueba unilateral (una sola cola) de Z. El nivel de significación fue de 0.05 . Se empleó el programa SPSS versión 19. Para el análisis estadístico descriptivo de las variables cuantitativas se utilizó media \pm desviación estándar y para las categóricas, frecuencias y porcentajes. La comparación de medias se realizó con t de Student y para la comparación de variables categóricas, la chi cuadrada de Pearson (univariado). Se estimó la razón de momios (RM) con intervalos de confianza de $95 \%$ (IC $95 \%$ ), con una $\mathrm{p}<0.05$.

\section{Resultados}

La muestra estuvo constituida por 200 sujetos, 100 casos y 100 controles. Los grupos fueron estratificados y pareados por edad y sexo. Al comparar las variables categóricas de los dos grupos se obtuvo una $\mathrm{p}=0.5$. La comparación de las variables continuas indicó una $\mathrm{p}=0.145$. La edad de los sujetos fue de $38.4 \pm 11.93$, rango entre 18 y 63 años; se trató de 151 mujeres $(75.5 \%)$ y 49 hombres $(24.5 \%)$. La comparación de las variables categóricas entre los dos grupos indicó una $p=0.5$. Al comparar las variables continuas se obtuvo una $\mathrm{p}=0.145$, que indicó que los grupos fueron similares. De acuerdo con las guías GINA se definieron tres grupos de control, pero para el análisis estadístico dicotómico se formó uno solo en el que se incluyó a los parcialmente controlados y mal controlados debido al pequeño tamaño de la muestra.

$\mathrm{Al}$ analizar la severidad del asma en el momento del diagnóstico, en 54 pacientes fue intermitente, en 35 leve persistente, en 14 moderada persistente $y$ en 97 grave persistente. Al estratificar por controlados y no controlados, 49 pacientes $(24.5 \%)$ con asma grave persistente fueron casos y 48 (24\%), controles; 25 pacientes (12.5\%) intermitentes fueron casos y $29(14.5 \%)$, controles. El total de los resultados se muestran en el cuadro 1 . Al estratificar por grupos etarios, 36 casos (18\%) se ubicaron entre 18 y 28 años y 33 controles (16.5 \%) entre 39 y 48 años, que constituyeron los grupos más representativos. Respecto a la escolaridad, 43 casos $(21.5 \%)$ y
30 controles $(15 \%)$ terminaron la preparatoria; 169 $(84.5 \%)$ residían en el sector urbano, 85 casos (42.5) y $84(42 \%)$ controles. Respecto al índice de masa corporal, 46 casos ( $23 \%$ ) se ubicaron como normales y solo 16 pacientes ( $8 \%$ ) presentaron obesidad; entre los controles, 56 pacientes $(28 \%)$ se identificaron en la categoría de sobrepeso $(p=0.01)$. Al analizar las comorbilidades asociadas con el asma, 85 casos (42.5\%) y 84 controles (42\%) presentaron rinitis alérgica $(\mathrm{p}=0.5)$. Los resultados completos del análisis de frecuencias y porcentajes entre casos $\mathrm{y}$ controles y sus respectivos valores de $\mathrm{p}$ se detallan en el cuadro 2.

En el grupo de los casos, 86 pacientes (43\%) fueron positivos a las pruebas cutáneas con alérgenos y 91 (45.5\%), negativos. Ni el valor de $\mathrm{p}$ $(\mathrm{p}=0.18)$ ni la RM $(1.64$, IC $95 \%=0.67-3.99)$ fueron estadísticamente significativos para ser factor de riesgo. En relación con el uso de inmunoterapia específica, el análisis indicó un resultado no significativo $(\mathrm{RM}=1)$.

Respecto a los valores de pruebas funcionales respiratorias, se tomó en cuenta el valor $\mathrm{VEF}_{1}$ medido en la última espirometría que tenía cada paciente, teniendo en cuenta que $<80 \%$ podría ser un factor de riesgo. Los pacientes con $\mathrm{VEF}_{1}>80 \%$ fueron 23 casos (11.5\%) y 53 controles (26.5). El RM en este grupo indicó que se trataba de un factor protector $(0.26$, IC $95 \%=0.14-0.48), \mathrm{p}=0.00$. El $47 \%$ de los casos (94 pacientes) y $46 \%$ de los controles (92 pacientes) señalaron el control médico estricto en su cita por consulta externa; no se observó asociación estadísticamente significativa $(\mathrm{RM}=1.36$, IC $95 \%=0.45-4.80)$. Se encontró asociación de protección cuando los pacientes utilizaron correctamente los inhaladores $(\mathrm{RM}=0.36$, IC $95 \%=0.13-0.97), p=0.031$. Los antecedentes familiares de atopia no mostraron asociación $(\mathrm{RM}=1.17$, IC $95 \%=0.67-2.05), \mathrm{p}=0.3$. En cuanto al recuento de eosinófilos absolutos en sangre periférica, los valores normales se constituyen como un factor protector; fueron normales en 48 casos ( $24 \%$ ) y 73 controles $(36.5 \%), R M=0.34$, IC $95 \%=0.18$ 0.61 y $\mathrm{p}=0.00$.

Estudiamos varias comorbilidades, los resultados completos se muestran en el cuadro 3. No hubo diferencia estadísticamente significativa como factor de riesgo de mal control de asma al analizar rinitis alérgica $(\mathrm{RM}=0.92$, IC $95 \%=0.43-1.99, \mathrm{p}=0.5)$, sinu- 


\begin{tabular}{|c|c|c|c|c|c|}
\hline \multirow{2}{*}{ Variables } & \multicolumn{2}{|c|}{ Casos* } & \multicolumn{2}{|c|}{ Controles ${ }^{* *}$} & \multirow[b]{2}{*}{$p$} \\
\hline & $\mathrm{n}$ & $\%$ & $\mathrm{n}$ & $\%$ & \\
\hline \multicolumn{5}{|l|}{ Edad (años) } & \multirow{5}{*}{0.145} \\
\hline $18-28$ & 36 & 18 & 20 & 10 & \\
\hline $29-38$ & 23 & 11.5 & 15 & 7.5 & \\
\hline $39-48$ & 25 & 12.5 & 33 & 16.5 & \\
\hline$>49$ & 16 & 8 & 32 & 16 & \\
\hline \multicolumn{5}{|l|}{ Sexo } & \multirow{3}{*}{0.5} \\
\hline Mujeres & 75 & 37.5 & 76 & 38 & \\
\hline Hombres & 25 & 12.5 & 24 & 12 & \\
\hline \multicolumn{5}{|l|}{ Estudios culminados } & \multirow{6}{*}{0.001} \\
\hline Ninguno & 6 & 3 & 2 & 1 & \\
\hline Primaria & 6 & 3 & 21 & 10.5 & \\
\hline Secundaria & 27 & 13.5 & 17 & 8.5 & \\
\hline Preparatoria & 43 & 21.5 & 30 & 15 & \\
\hline Superior & 18 & 9 & 30 & 15 & \\
\hline \multicolumn{5}{|l|}{ Lugar de vivienda } & \multirow{3}{*}{0.5} \\
\hline Urbana & 85 & 42.5 & 84 & 42 & \\
\hline Rural & 15 & 7.5 & 16 & 8 & \\
\hline \multicolumn{5}{|l|}{ Ingresos económicos } & \multirow{5}{*}{0.66} \\
\hline Muy bajos & 17 & 8.5 & 14 & 7 & \\
\hline Bajos & 22 & 11 & 29 & 14.5 & \\
\hline Medios & 43 & 21.5 & 38 & 19 & \\
\hline Altos & 18 & 9 & 19 & 9.5 & \\
\hline \multicolumn{5}{|l|}{ Índice de masa corporal } & \multirow{5}{*}{0.01} \\
\hline Peso bajo & 0 & 0 & 0 & 0 & \\
\hline Normal & 46 & 23 & 26 & 13 & \\
\hline Sobrepeso & 38 & 19 & 56 & 28 & \\
\hline Obesidad & 16 & 8 & 18 & 9 & \\
\hline \multicolumn{5}{|c|}{ Clasificación por severidad del asma al momento del diagnóstico } & \multirow{5}{*}{0.72} \\
\hline Intermitente & 25 & 12.5 & 29 & 14.5 & \\
\hline $\begin{array}{l}\text { Leve } \\
\text { persistente }\end{array}$ & 20 & 10 & 15 & 7.5 & \\
\hline Moderado persistente & 6 & 3 & 8 & 4 & \\
\hline $\begin{array}{l}\text { Grave } \\
\text { persistente }\end{array}$ & 49 & 24.5 & 48 & 24 & \\
\hline \multicolumn{5}{|l|}{ Adherencia al tratamiento } & \multirow{7}{*}{0.81} \\
\hline Nunca se olvida & 61 & 30.5 & 60 & 30 & \\
\hline Se olvida 1 vez/semana & 15 & 7.5 & 13 & 6.5 & \\
\hline Se olvida $2-3 /$ mes & 16 & 8 & 22 & 11 & \\
\hline Se olvida $2-3 / 3$ meses & 4 & 2 & 3 & 1.5 & \\
\hline Se olvida $2-3 / 6$ meses & 3 & 1.5 & 1 & 0.5 & \\
\hline Se olvida 2-3/año & 1 & 0.5 & 1 & 0.5 & \\
\hline
\end{tabular}


sitis $(\mathrm{RM}=1.43$, IC $95 \%=0.76-2.70, \mathrm{p}=0.33)$, poliposis nasosinusal $(\mathrm{RM}=1.58$, IC $95 \%=0.67-3.72$, $\mathrm{p}=0.19)$ y la enfermedad por reflujogastroesofágico, $\operatorname{ERGE}(\mathrm{RM}=1$, IC $95 \%=0.55-1.81, \mathrm{p}=0.55)$. Dos comorbilidades presentaron asociación estadísticamente significativa: la dermatitis atópica fue un factor de riesgo, se presentó en 11 casos $(5.5 \%)$ y 22 controles $(11 \%)$ y arrojó un $\mathrm{RM}=2.28$, IC $95 \%=$ 1.04-5.00, $\mathrm{p}=0.02$; la ausencia de estrés fue un factor de protección y se identificó en 27 casos (13.5\%) y 47 controles $(23.5 \%), \mathrm{RM}=0.41$, IC $95 \%=0.23$ $0.75, \mathrm{p}=0.03)$.
Se analizaron varias condiciones ambientales sin que alguna representara un factor de riesgo, como el uso de aire acondicionado en el hogar o en el trabajo, la presencia de mascotas en el hogar y el uso de medidas antiácaros. El tabaquismo actual y activo se identificó como factor de riesgo $(\mathrm{RM}=4.23$ IC $95 \%=1.35-13.2, p=0.007)$, sin embargo, el tabaquismo pasivo no demostró ser factor de riesgo $(\mathrm{RM}=0.85, \mathrm{IC} 95 \%=0.44-1.62, \mathrm{p}=0.37)$. Manifestaron no tener alfombras o peluches, 68 casos (34\%) y 84 controles $(42 \%), \mathrm{RM}=0.4$, IC $95 \%=0.2-0.79$, $\mathrm{p}=0.04$, lo que indicó que es un factor protector.

\begin{tabular}{|c|c|c|c|c|c|c|c|}
\hline \multirow{2}{*}{ Variables } & \multicolumn{2}{|c|}{ Casos } & \multicolumn{2}{|c|}{ Controles } & \multirow{2}{*}{ RM } & \multirow{2}{*}{ IC $95 \%$} & \multirow{2}{*}{$p$} \\
\hline & $\mathrm{n}$ & $\%$ & $\mathrm{n}$ & $\%$ & & & \\
\hline \multicolumn{5}{|c|}{ Pruebas cutáneas } & \multirow{3}{*}{1.64} & \multirow{3}{*}{$0.67-3.99$} & \multirow{3}{*}{0.18} \\
\hline Positivas & 86 & 43 & 91 & 45.5 & & & \\
\hline Negativas & 14 & 7 & 9 & 4.5 & & & \\
\hline \multicolumn{5}{|c|}{ Inmunoterapia específica } & \multirow{3}{*}{1} & \multirow{3}{*}{$0.52-1.89$} & \multirow{3}{*}{0.56} \\
\hline Sí & 75 & 37.5 & 75 & 37.5 & & & \\
\hline No & 25 & 12.5 & 25 & 12.5 & & & \\
\hline \multicolumn{5}{|c|}{ Último $V_{E F}$ medido } & \multirow{3}{*}{0.26} & \multirow{3}{*}{$0.14-0.48$} & \multirow{3}{*}{0.00} \\
\hline$<80 \%$ & 77 & 38.5 & 47 & 23.5 & & & \\
\hline$>80 \%$ & 23 & 11.5 & 53 & 26.5 & & & \\
\hline \multicolumn{5}{|c|}{ Control médico ordenado } & \multirow{3}{*}{1.36} & \multirow{3}{*}{$0.45-4.08$} & \multirow{3}{*}{0.31} \\
\hline Sí & 94 & 47 & 92 & 46 & & & \\
\hline No & 6 & 3 & 8 & 4 & & & \\
\hline \multicolumn{5}{|c|}{ Uso correcto de los inhaladores } & \multirow{3}{*}{0.36} & \multirow{3}{*}{$0.13-0.97$} & \multirow{3}{*}{0.031} \\
\hline Sí & 85 & 42.5 & 94 & 47 & & & \\
\hline No & 15 & 7.5 & 6 & 3 & & & \\
\hline \multicolumn{5}{|c|}{ Antecedente de atopia en la familia } & \multirow{3}{*}{1.17} & \multirow{3}{*}{$0.67-2.05$} & \multirow{3}{*}{0.33} \\
\hline Sí & 43 & 21.5 & 47 & 23.5 & & & \\
\hline No & 57 & 28.5 & 53 & 26.5 & & & \\
\hline \multicolumn{5}{|c|}{ IgE sérica total } & \multirow{3}{*}{0.81} & & \\
\hline Normal & 44 & 22 & 49 & 24.5 & & $0.46-1.42$ & 0.28 \\
\hline Elevado & 56 & 28 & 51 & 25.5 & & & \\
\hline Eosinófilos en & & & & & & & \\
\hline Normal & 48 & 24 & 73 & 36.5 & 0.34 & $0.18-0.61$ & 0.0 \\
\hline Elevado & 52 & 26 & 27 & 13.5 & & & \\
\hline $\begin{array}{l}{ }^{\star} \text { Casos }=\text { pacient } \\
{ }^{\star *} \text { Controles }=\text { pac }\end{array}$ & $\begin{array}{l}\text { te con } \\
\text { introla }\end{array}$ & mal con & & & & & \\
\hline
\end{tabular}




\section{Discusión}

En este estudio se demuestra que las variables asociadas con el mal control del asma en la población entre 18 y 63 años son el tabaquismo actual y activo y la presencia de dermatitis atópica. Mientras que el recuento normal de eosinófilos en sangre, el uso correcto y apropiado de los inhaladores, el $\mathrm{VEF}_{1}$ $>80 \%$, la ausencia de alfombras o peluches y la no presencia de estrés se comportan como factores protectores para el mal control del asma.

Uno de los hallazgos más interesantes fue que el riesgo de presentar mal control del asma disminuye con la edad de los pacientes. Este dato está acorde con los que se desprenden de estudios previos, como los llevados a cabo en el Reino Unido y Australia, que efectuaron un seguimiento hasta la edad de 33 y 35 años, respectivamente, de niños que habían comenzado a padecer asma en edades tempranas de la vida. ${ }^{15}$

Según los resultados de nuestro trabajo, cuanto mayor es el nivel cultural del paciente, mayor riesgo de asma. Este dato llama la atención porque diversos estudios reportan lo contrario, ${ }^{16}$ lo que se explica por el número reducido de pacientes sin estudio o estudios básicos. En este sentido se ha argumentado que el menor estatus socioeconómico y el bajo nivel cultural podrían explicar la mayor prevalencia de asma en ciertos grupos poblacionales, ${ }^{17}$ sin embargo, en la actualidad no hay datos consistentes que establezcan un nexo claro entre el riesgo de padecer asma y el nivel socioeconómico. ${ }^{17}$ Un parámetro objetivo, medible y reproducible para estadificar la condición socioeconómica es el ingreso mensual. Nuestro estudio demuestra que la mayor parte de los pacientes se ubican en nivel socioeconómico medio, lo que demuestra que la pobreza está asociada con mal control del asma.

La sensibilización a alérgenos parece tener una clara relación con el asma en adultos. Los aeroalérgenos procedentes de los perros y gatos se asocian con partículas de menor tamaño que otras fuentes alergénicas, lo que facilita su penetración en las vías aéreas inferiores y aumenta su potencial patogénico. ${ }^{18}$ Paradójicamente, la presencia de animales en el domicilio no parece relacionarse con el mal control del asma, lo que concuerda con la información realizada acerca de la inocuidad del epitelio de perro o incluso su papel protector.

Este estudio analiza no solo sensibilización a perros y gatos, sino a los aeroalérgenos; las pruebas cutáneas positivas no fueron estadísticamente significativas y se presentaron en 86 casos (43\%) y 91 controles $(45.5 \%)$, si bien tuvieron $\mathrm{RM}=1.64 \mathrm{e}$ IC $95 \%=0.67-3.99$. En el caso de los gatos, los resultados hasta el momento han sido más dispares. ${ }^{19}$ La presencia de mascotas en el hogar presentó $\mathrm{RM}=0.73$, IC $95 \%=0.4-1.31, \mathrm{p}=0.18$, por 10 tanto no tuvo significación estadística.

En los sujetos evaluados se notó alta incidencia de rinitis, no así de poliposis nasal. Estudios prospectivos de 20 años de seguimiento permiten afirmar que la rinitis es un factor de riesgo para el desarrollo y la gravedad del asma. Diferentes análisis muestran que ambas entidades a menudo coexisten en el mismo paciente: la rinitis aparece en $75 \%$ de los pacientes con asma alérgica y en $80 \%$ de aquellos con asma no alérgica. ${ }^{20}$ En nuestra investigación, la frecuencia de rinitis alérgica fue de 85 sujetos entre los casos $(42.5 \%)$, sin embargo, en los pacientes bien controlados se presentó en 84 (42\%), por lo que no constituyó un factor de riesgo. Un estudio multicéntrico español sobre la coexistencia de rinitis y asma corroboró la alta prevalencia de asociación en pacientes alérgicos: afectó a $89.5 \%$ de 942 incluidos. ${ }^{21}$ En esta muestra de 200 asmáticos estudiados, 169 $(84.5 \%)$ tuvieron rinitis alérgica.

Queda en duda la importancia de los antecedentes familiares como factor de riesgo para asma mal controlada en el adulto. Si bien en la actualidad esta tendencia a la agregación familiar deja poco lugar a la duda, ${ }^{22}$ los intentos por establecer un patrón de herencia han sido infructuosos, probablemente debido a que el asma bronquial puede ser la expresión fenotípica de diferentes genotipos. ${ }^{23}$

Respecto a la exposición a ciertos agentes irritantes del medio ambiente, el estudio PRESS ha demostrado que el tabaquismo actual y activo es un factor de riesgo para el desarrollo del mal control del asma en adultos $(\mathrm{RM}=4.23$, IC $95 \%=1.35-13.2$, $\mathrm{p}=0.007$ ). Aunque en nuestro estudio el tabaquismo pasivo no ha mostrado diferencias estadísticamente significativas entre casos y controles, es conocida la mayor severidad del asma en pacientes fumadores pasivos, comparados con los que no tienen esta exposición. $^{24}$

La relación entre el asma del adulto y la presencia de alfombras o peluches es bien conocida. Entre 5 y $10 \%$ de los adultos asmáticos presenta exacerbaciones frecuentes cuando tienen contacto constante 
Fernández de Córdova-Aguirre JC et al. Mal control de asma

\begin{tabular}{|c|c|c|c|c|c|c|c|}
\hline \multirow{2}{*}{ Variables } & \multicolumn{2}{|c|}{ Casos } & \multicolumn{2}{|c|}{ Controles } & \multirow{2}{*}{ RM } & \multirow{2}{*}{ IC $95 \%$} & \multirow{2}{*}{$p$} \\
\hline & $\mathrm{n}$ & $\%$ & $\mathrm{n}$ & $\%$ & & & \\
\hline \multicolumn{8}{|c|}{ Comorbilidades } \\
\hline \multicolumn{8}{|c|}{ Rinitis alérgica } \\
\hline Sí & 85 & 42.5 & 84 & 42 & \multirow[t]{2}{*}{0.92} & \multirow[t]{2}{*}{ 0.43-1.99 } & \multirow[t]{2}{*}{0.5} \\
\hline No & 15 & 7.5 & 16 & 8 & & & \\
\hline \multicolumn{5}{|l|}{ Sinusitis } & \multirow{3}{*}{1.43} & \multirow{3}{*}{$0.76-2.70$} & \multirow{3}{*}{0.33} \\
\hline Si & 23 & 11.5 & 30 & 15 & & & \\
\hline No & 77 & 38.5 & 70 & 35 & & & \\
\hline \multicolumn{5}{|c|}{ Poliposis nasal } & \multirow{3}{*}{1.58} & \multirow{3}{*}{$0.67-3.72$} & \multirow{3}{*}{0.19} \\
\hline Sí & 10 & 5 & 15 & 7.5 & & & \\
\hline No & 90 & 45 & 85 & 42.5 & & & \\
\hline \multicolumn{5}{|c|}{ Enfermedad por reflujogastroesofágico } & \multirow{3}{*}{1} & \multirow{3}{*}{$0.55-1.81$} & \multirow{3}{*}{0.56} \\
\hline Sí & 32 & 16 & 32 & 16 & & & \\
\hline No & 68 & 34 & 68 & 34 & & & \\
\hline \multicolumn{5}{|l|}{ Estrés } & \multirow{3}{*}{0.41} & & \\
\hline Sí & 73 & 36.5 & 53 & 26.5 & & $0.23-0.75$ & 0.03 \\
\hline No & 27 & 13.5 & 47 & 23.5 & & & \\
\hline Dermatiti & & & & & & & \\
\hline Sí & 11 & 5.5 & 22 & 11 & 2.28 & $1.04-5.0$ & 0.02 \\
\hline No & 89 & 44.5 & 78 & 39 & & & \\
\hline Condicio & & & & & & & \\
\hline Aire acon & & & & & & & \\
\hline Sí & 12 & 6 & 19 & 9.5 & 1.72 & $0.78-3.76$ & 0.12 \\
\hline No & 88 & 44 & 81 & 44.5 & & & \\
\hline Tabaquisn & & & & & & & \\
\hline Sí & 4 & 2 & 15 & 7.5 & 4.23 & $1.35-13.2$ & 0.007 \\
\hline No & 96 & 48 & 85 & 42.5 & & & \\
\hline Tabaquisn & & & & & & & \\
\hline Sí & 26 & 13 & 23 & 11.5 & 0.85 & $0.44-1.62$ & 0.37 \\
\hline No & 74 & 37 & 77 & 38.5 & & & \\
\hline Mascotas & & & & & & & \\
\hline Sí & 69 & 39.5 & 62 & 31 & 0.73 & $0.4-1.31$ & 0.18 \\
\hline No & 31 & 15.5 & 38 & 19 & & & \\
\hline Medidas & & & & & & & \\
\hline Sí & 32 & 16 & 24 & 12 & 1.49 & $0.8-2.77$ & 0.135 \\
\hline No & 68 & 34 & 76 & 38 & & & \\
\hline Alfombras & & & & & & & \\
\hline Sí & 32 & 16 & 16 & 8 & 0.4 & $0.2-0.79$ & 0.04 \\
\hline No & 68 & 34 & 84 & 42 & & & \\
\hline $\begin{array}{l}{ }^{*} \text { Casos }=\mathrm{pa} \\
{ }^{*} \text { Controles }\end{array}$ & $\begin{array}{l}\text { nte co } \\
\text { ontrol }\end{array}$ & s y mal c & dos. & & & & \\
\hline
\end{tabular}


con alfombras o peluches, por lo tanto, evitar estos factores de riesgo son factores de protección. Nuestro hallazgo corrobora esta misma relación. ${ }^{25}$

De acuerdo con estudios previos se constató que la dermatitis atópica puede exacerbar el asma bronquial. Se sabe que tiene la capacidad de incrementar el grado de hiperreactividad bronquial por inflamación tipo TH2 y producir diversas alteraciones en las vías aéreas que comprometen el mantenimiento de su función. ${ }^{26}$

Aunque la obesidad está estrechamente relacionada, en nuestro estudio no se observaron diferencias estadísticamente significativas entre los casos y los controles al respecto. Este hecho contrasta con los resultados obtenidos por la National Asthma Survey ${ }^{27}$ en cuanto al nivel de sedentarismo y sobrepeso u obesidad y su influencia en el asma, que establece una relación entre la obesidad y la severidad del asma en los adultos norteamericanos que la padecen. En este sentido, Koh et al. realizaron un estudio multicéntrico en una población de 368 adolescentes entre 12 y 20 años con asma moderada severa; observaron que la adiposidad se asoció con peor control del asma en mujeres y se evidenció un mejor control de la enfermedad en los varones, por influjo de la adiponectina. ${ }^{28}$

La mayoría de los pacientes mal controlados se ubicaron al momento del diagnóstico en el grupo de asma grave persistente. Aunque este grado de severidad estadísticamente no demostró ser un factor de riesgo, por la alta frecuencia de presentación es un factor muy importante a tomar en cuenta. Esto orienta a que el diagnóstico y tratamiento tempranos de la enfermedad son importantes antes de que se presenten características de gravedad. El ERGE es un disparador de los síntomas de asma y puede favorecer la sensibilización a proteínas de algunos alimentos al incrementar el tiempo de exposición del antígeno. El asma y el ERGE son dos entidades comunes que pueden coexistir sin una interacción directa. El ERGE entre los pacientes con asma se estima entre 33 y $89 \%$; puede estar presente sin síntomas esofágicos y en ocasiones solo puede ocasionar aumento en el número de ventilaciones por minuto. Muchos pacientes con sibilancias y tos crónica tienen ERGE subyacente. Es necesaria una medición ambulatoria de 24 horas del $\mathrm{pH}$ esofágico para identificar ERGE clínicamente silenciosa, sin embargo, todavía no están bien establecidos los mecanismos por los cuales estos factores dirigen la producción de citocinas $\mathrm{Th} 2$, lo que abre un panorama muy amplio para la investigación. ${ }^{29}$ En el presente análisis no demostró ser un factor de riesgo.

Este estudio posee algunas limitaciones que deben tomarse en cuenta. Dado que se eligieron pacientes mayores de 18 años, no se evaluó un porcentaje elevado de pacientes asmáticos (adolescentes), por lo que las conclusiones obtenidas solo pueden extrapolarse a este grupo de edad. También el hecho de que los sujetos no se sometieran a la prueba de provocación bronquial con metacolina o histamina pudo hacer incurrir en un sesgo de selección, dado que pacientes clasificados como no asmáticos podrían serlo. En conclusión, existen diferentes variables asociadas con el mal control del asma en la edad adulta. El conocimiento de estas puede ayudar a prevenir la aparición de exacerbaciones y mala calidad de vida en dicha población.

\section{Referencias}

1. Prosser R, Carleton B, Smith A. The comorbidity burden of the treated asthma patient population in British Columbia. Chronic Dis Can. 2010;30(2):46-55. Disponible en: https://www.canada.ca/en/public-health/ services/reports-publications/health-promotion-chronic-disease-prevention-canada-research-policypractice/vol-30-no-2-2010/comorbidity-burden-treated-asthma-patient-population-british-columbia.html

2. Platts-Mills TA, Erwin EA, Heymann PW, Woodfolk JA. Pro: the evidence for a causal role of dust mites in asthma. Am J Respir Crit Care Med. 2009;180(2):109-121. DOI: 10.1164/rccm.200811-1756PR

3. Tovey E, Ferro A. Time for new methods for avoidance of house dust mite and other allergens. Curr Allergy Asthma Rep. 2012;12(5):465-477. DOI: 10.1007/s11882-012-0285-0

4. Gershon AS, Wang C, Guan J, To T. Burden of comorbidity in individuals with asthma. Thorax. 2010;65(7):612-618. DOI: 10.1136/thx.2009.131078

5. Global Strategy for Asthma Management and Prevention. EE. UU.: Global Initiative for Asthma (GINA); 2018. Disponible en: https://ginasthma.org/wp-content/uploads/2018/04/wms-GINA-2018-report-V1.3-002.pdf 
6. National Asthma Education and Prevention Program. Expert Panel Report 3. Guidelines for the diagnosis and management of asthma. EE. UU.: Department of Health and Human Services/National Institutes of Health/National Heart, Lung, and Blood Institute; 2007. Disponible en: https://www.nhlbi.nih.gov/files/ docs/guidelines/asthgdln.pdf

7. British Thoracic Society/Scottish Intercollegiate Guidelines Network. British Guideline on the Management of Asthma. Escocia: British Thoracic Society/Scottish Intercollegiate Guidelines Network; 2008. Disponible en: https://www.brit-thoracic.org.uk/document-library/clinical-information/asthma/ btssign-asthma-guideline-2008/

8. Cazzola M, Calzetta L, Bettoncelli G, Novelli L, Cricelli C, Rogliani P, et al. Asthma and comorbid medical illness. Eur Respir J. 2011;38(1):42-49. DOI: 10.1183/09031936.00140310

9. Antunes J, Fernandes A, Miguel-Borrego L, Leiria-Pinto P, Cavaco J. Cystic fibrosis, atopy, asthma and ABPA. Allergol Immunopathol (Madr). 2010;38(5):278-284. DOI: 10.1016/j.aller.2010.06.002

10. Vandenplas O, Dramaix M, Joos G, Louis R, Michils A, Verleden G, et al. The impact of concomitant rhinitis on asthma-related quality of life and asthma control. Allergy. 2010;65(10):1290-1297. DOI: 10.1111/j.1398-9995.2010.02365.x

11. Thilsing T, Rasmussen J, Lange B, Kjeldsen AD, Al-Kalemji A, Baelum J. Chronic rhinosinusitis and occupational risk factors among 20- to 75-year-old Danes: a GA2LEN-based study. Am J Ind Med. 2012;55(11):1037-1043. DOI: 10.1002/ajim.22074

12. Jarvis D, Newson R, Lotvall J, Hastan D, Tomassen P, Keil T, et al. Asthma in adults and its association with chronic rhinosinusitis: the GA2LEN survey in Europe. Allergy. 2012;67(1):91-98. DOI: 10.1111/j.13989995.2011.02709.x

13. Franco-Marina F, Fernández-Plata R, Torre-Bouscoulet L, García-Sancho C, Sánchez-Gallen E, Martínez D, Pérez-Padilla R. Efficient screening for COPD using three steps: a cross-sectional study in Mexico City. NPJ Prim Care Respir Med. 2014;24:14002. DOI: 10.1038/npjpcrm.2014.2

14. Abramson MJ, Puy RM, Weiner JM. Injection allergen immunotherapy for asthma. Cochrane Database Syst Rev. 2010;(8):CD001186. DOI: 10.1002/14651858.CD001186.pub2

15. Chipps BE, Zeiger RS, Borish L, Wenzel SE, Yegin A, Hayden ML, et al. Key findings and clinical implications from The Epidemiology and Natural History of Asthma: Outcomes and Treatment Regimens (TENOR) study. J Allergy Clin Immunol. 2012;130(2):332-342. DOI: 10.1016/j.jaci.2012.04.014

16. Harris M, Lundien M, Finnie D, Williams A, Beebe T, Sloan J, et al. Application of a novel socioeconomic measure using individual housing data in asthma research: an exploratory study. NPJ Prim Care Respir Med. 2014;24:14018. DOI: 10.1038/npjpcrm.2014.18

17. Halimi L, Pry R, Pithon G, Godard P, Varrin M, Chanez P. Severe asthma and adherence to peak flow monitoring: longitudinal assessment of psychological aspects. J Psychosom Res. 2010;69(4):331-340. DOI: 10.1016/j.jpsychores.2010.02.007

18. Just J, Gouvis-Echraghi R, Couderc R, Guillemot-Lambert N, Saint-Pierre P. Novel severe wheezy young children phenotypes: boys atopic multiple-trigger and girls nonatopic uncontrolled wheeze. J Allergy Clin Immunol. 2012;130(1):103-110. DOI: 10.1016/j.jaci.2012.02.041

19. Sánchez J, Diez S, Cardona R. Frequency of sensitization to animals in a tropical area. Rev Alerg Mex. 2014;61(2):81-89. DOI: 10.29262/ram.v61i2.30

20. Brozek JL, Bousquet J, Baena-Cagnani CE, Bonini S, Canonica GW, Casale TB, et al. Allergic Rhinitis and its Impact on Asthma (ARIA) guidelines: 2010 revision. J Allergy Clin Immunol. 2010;126(3):466476. DOI: 10.1016/j.jaci.2010.06.047

21. Bousquet J, Addis A, Adcock I, Agache I, Agusti A, Alonso A, et al. Integrated care pathways for airway diseases (AIRWAYS-ICPs). Eur Respir J. 2014;44(2):304-323. DOI: 10.1183/09031936.00014614

22. Portelli MA, Hodge E, Sayers I. Genetic risk factors for the development of allergic disease identified by genome wide association. Clin Exp Allergy. 2014;45(1):21-31. DOI: 10.1111/cea.12327

23. Haldar P, Pavord ID, Shaw DE, Berry MA, Thomas M, Brightling CE, et al. Cluster analysis and clinical asthma phenotypes. Am J Respir Crit Care Med. 2008;178(3):218-224. DOI: 10.1164/rccm.200711-1754OC 
24. Amelink M, De Nijs SB, Berger M, Weersink EJ, Ten-Brinke A, Sterk PJ, et al. Nonatopic males with adult onset asthma are at risk of persistent airflow limitation. Clin Exp Allergy. 2012;42(5):769-774. DOI: 10.1111/j.1365-2222.2012.03977.x

25. Reponen T, Levin L, Zheng S, Vesper S, Ryan P, Grinshpun SA, LeMasters G. Family and home characteristics correlate with mold in homes. Environ Res. 2013;124:67-70. DOI: 10.1016/j. envres.2013.04.003

26. Eichenfield LF, Tom WL, Chamlin SL, Feldman SR, Hanifin JM, Simpson EL, et al. Guidelines of care for the management of atopic dermatitis: section 1. diagnosis and assessment of atopic dermatitis. J Am Acad Dermatol. 2014;70(2):338-351. DOI: 10.1016/j.jaad.2013.10.010

27. Lai CK, Beasley R, Crane J, Foliaki S, Shah J, Weiland S, et al. Thorax. 2009;64(6):476-483. DOI: 10.1136/thx.2008.106609

28. Koh YY, Kim CK. Desarrollo de asma en pacientes con rinitis alérgica. Curr Opin Allergy Clin Immunol. 2003;3(3):159-164.

29. Reddel HK, Taylor DR, Bateman ED, Boulet LP, Boushey HA, Busse WW, et al. An official American Thoracic Society/European Respiratory Society statement: asthma control and exacerbations: standardizing endpoints for clinical asthma trials and clinical practice. Am J Respir Crit Care Med. 2009;180(1):59-99. DOI: 10.1164/rccm.200801-060ST 